УДК 621.315 .592

\title{
Влияют ли химические эффекты на накопление структурных нарушений при имплантации в GaN ионов фтора?
}

\author{
(C) А.И. Титов, К.В. Карабешкин, П.А. Карасев, А.И. Стручков \\ Санкт-Петербургский политехнический университет Петра Великого, \\ 195251 Санкт-Петербург, Россия \\ E-mail: andrei.titov@rphf.spbstu.ru
}

Поступила в Редакцию 22 апреля 2019 г.

В окончательной редакции 28 апреля 2019 г.

Принята к публикации 28 апреля 2019 г.

Исследовано накопление структурных повреждений в $\mathrm{GaN}$ при облучении ускоренными ионами $\mathrm{F}$ и $\mathrm{Ne}$ с энергиями 1.3 и 3.2 кэВ/а.е.м. Показано, что в рамках рассматриваемых доз химические эффекты при внедрении ионов фтора не оказывают существенного влияния на образование устойчивых структурных повреждений как в объеме, так и на поверхности $\mathrm{GaN}$.

Ключевые слова: GaN, ионное облучение, инженерия дефектов, химические эффекты.

DOI: $10.21883 /$ FTP.2019.11.48439.9145

\section{1. Введение}

Имплантация ионов является одним из основных методов, которые используются для введения легирующей примеси при создании полупроводниковых приборов. Хорошо известно, что бомбардировка полупроводников ионами всегда сопровождается образованием устойчивых радиационных дефектов [1], которое может усиливаться в результате химических эффектов. В частности, как отмечали авторы [2,3] для случая ионного облучения нитрида галлия, все рассматриваемые ими легкие ионы $(\mathrm{C}, \mathrm{O}$, и $\mathrm{Si})$ усиливали накопление разупорядочения в ходе имплантации. В то же время нам не известны специальные публикации, посвященные влиянию химических эффектов при внедрении ионов промежуточной массы - ионов фтора - на эффективность радиационного повреждения GaN. Хотя F это самый активный неметалл, который эффективно взаимодействует почти со всеми веществами и образует соединения практически со всеми химическими элементами. В частности, ранее [4] для объяснения эффектов сильного увеличения поверхностной шероховатости и свеллинга при облучении $\mathrm{GaN}$ ионами фтора среди прочего предполагалось и влияние химических эффектов при подобном воздействии. Заметим, что имеются свидетельства того, что при имплантации ионов фтора в монокристаллический кремний происходит аномальное дефектообразование [5].

Усиление образования структурных нарушений может происходить по нескольким причинам [6]: из-за захвата мигрирующих точечных дефектов внедренными атомами примеси; в результате формирования новой фазы и возникновения деформации кристаллической структуры; наконец, из-за повышения стабильности комплексов дефектов в областях с повышенной концентрацией примеси.

Данная работа посвящена установлению роли химических эффектов в накоплении структурных нарушений в нитриде галлия при имплантации в него ионов фтора. Для этого наряду с ионами фтора в образцы внедрялись и ионы неона. Действительно, с одной стороны, масса и атомный номер у $\mathrm{F}$ и $\mathrm{Ne}$ почти совпадают, а с другой - их химические свойства существенным образом отличаются, и при наличии химических эффектов можно ожидать проявление заметных различий при имплантации неона и фтора в $\mathrm{GaN}$.

\section{2. Методика эксперимента}

Для корректного сравнения эффективности накопления повреждений при облучении ионами $\mathrm{F}$ и $\mathrm{Ne}$, единственным различием должны быть химические свойства имплантируемых атомов. Для этого условия облучения должны быть одинаковыми, т.е. распределение по глубине скорости образования первичных дефектов для обоих типов ионов должно совпадать. Другими словами, энергии ионов $\mathrm{F}$ и $\mathrm{Ne}$ должны быть подобраны таким образом, чтобы положения максимумов функций генерации атомных смещений были одинаковы, и плотности потока ионов, выраженные в единицах DPA/c, также совпадали. Здесь DPA (Displacements per Atom) - число атомных смещений в пересчете на атом мишени в максимуме профиля упругих потерь энергии иона.

B настоящей работе величины DPA были вычислены с помощью кода TRIM (версия SRIM-2013.00) [7] с эффективной пороговой энергией атомных смещений 25 эВ для подрешеток $\mathrm{Ga}$ и N. Концентрация атомов в $\mathrm{GaN}$ принималась за $8.85 \cdot 10^{22} \mathrm{~cm}^{-3}$. Результаты облучения сравнивались для одинаковых доз, выраженных в единицах DPA.

Легированные кремнием эпитаксиальные слои $n$-GaN со структурой вюрцита (0001), выращенные на сапфировой подложке по технологии MOVPE во ФТИ им. Иоффе (Санкт-Петербург, Россия), толщиной 2 мкм, облуча- 
Энергии, плотности потока и дозы ионов, использованные при облучении GaN, а также величина дозы 1 DPA. Данные по облучению ионами С взяты из [2]

\begin{tabular}{|c|c|c|c|c|c|c|}
\hline \multirow{2}{*}{ Ион } & \multirow{2}{*}{$\begin{array}{c}\text { Энергия } \\
\text { кэВ }\end{array}$} & \multicolumn{2}{|c|}{ Плотность потока ионов } & \multicolumn{2}{|c|}{ Доза ионов } & \multirow{2}{*}{$\begin{array}{c}1 \mathrm{DPA} \\
10^{15} \mathrm{~cm}^{-2}\end{array}$} \\
\hline & & $10^{12} \mathrm{~cm}^{-2} \cdot \mathrm{c}^{-1}$ & $\mathrm{DPA} \cdot \mathrm{c}^{-1}$ & $10^{15} \mathrm{~cm}^{-2}$ & DPA & \\
\hline $\mathrm{F}$ & $\begin{array}{l}25 \\
61\end{array}$ & $\begin{array}{l}3.67 \\
4.32\end{array}$ & $\begin{array}{l}3.6 \\
3.6\end{array}$ & $\begin{array}{c}2.55-20.4 \\
3-24\end{array}$ & $\begin{array}{l}2.5-20 \\
2.5-20\end{array}$ & $\begin{array}{l}1.02 \\
1.2\end{array}$ \\
\hline $\mathrm{Ne}$ & $\begin{array}{l}26 \\
64\end{array}$ & $\begin{array}{l}3.31 \\
3.76\end{array}$ & $\begin{array}{l}3.6 \\
3.6\end{array}$ & $\begin{array}{r}2.3-18.4 \\
2.61-20.9\end{array}$ & $\begin{array}{l}2.5-20 \\
10-20\end{array}$ & $\begin{array}{l}0.92 \\
1.045\end{array}$ \\
\hline $\mathrm{C}$ & 40 & 14 & 6.45 & $1-60$ & $0.46-27.6$ & 2.17 \\
\hline
\end{tabular}

лись при комнатной температуре ионами с энергиями 1.3 и 3.2 кэВ/а.е.м. Условия облучения представлены в таблице.

Условия для корректного сравнения результатов, о которых говорилось выше, полностью выполнялись. Это хорошо видно из рис. 1, где показаны вычисленные с помощью программы TRIM [7] распределения по глубине скорости объемной генерации атомных смещений для используемой плотности потока ионов для ионов неона и фтора с соответствующими энергиями.

Разупорядочение, возникшее при имплантации, измерялось ex situ методом резерфордовского обратного рассеяния с каналированием $(\mathrm{RBS} / \mathrm{C})$ ионов $0.7 \mathrm{MэB}{ }^{4} \mathrm{He}^{++}$, падающих по направлению [0001] и рассеиваемых на угол $103^{\circ}$ для улучшения разрешения по глубине. Как имплантация, так и анализ $\mathrm{RBS} / \mathrm{C}$ проводились на имплантере $500 \mathrm{kV}$ HVEE.

Спектры RBS/C для получения распределений по глубине эффективного числа рассеивающих центров, называемого в дальнейшем „относительным разупорядочением“, анализировались с помощью одного из стандартных алгоритмов [8].

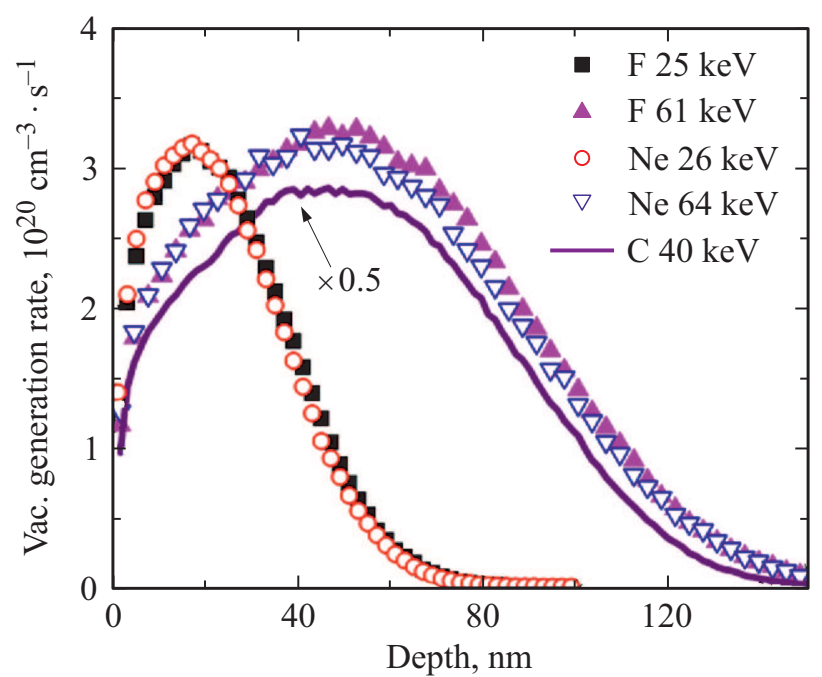

Рис. 1. Распределение по глубине $\mathrm{GaN}$ усредненной скорости генерации вакансий ионами $\mathrm{C}, \mathrm{F}$ и $\mathrm{Ne}$ с энергиями, указанными на вставке. Плотность потока ионов см. в таблице. Для ионов С 40 кэВ профиль приведен в половинном масштабе.

\section{3. Экспериментальные результаты и обсуждение}

На рис. 2 в качестве примеров показаны профили распределения по глубине относительного разупорядочения для нескольких эквивалентных доз, выраженных в DPA, для ионов фтора и неона с соответствующими энергиями. Заметим, что полученные распределения структурных нарушений имеют стандартный вид для подобного рода зависимостей, представленных в более ранних публикациях [2,3,9-11]: бимодальные функции с одним максимумом, расположенным на поверхности (поверхностный максимум дефектов ПМД), и вторым максимумом в объеме (ОМД). Еще раз подчеркнем, что условия генерации первичных дефектов в рассматриваемых экспериментах совершенно идентичны. Совпадает и полное число первичных атомных смещений, созданных за время накопления каждой дозы. Таким образом, единственной разницей при имплантации ионов в данных условиях являются только химические свойства внедряемых частиц. Поскольку распределения дефектов по глубине, как видно из рисунков, оказались совпадающими в пределах погрешности эксперимента, которая составляла около $10 \%$, можно сделать заключение, что эти свойства при рассматриваемых условиях не влияют заметным образом на формирование устойчивых структурных нарушений.

Проверим справедливость этого заключения, проследив зависимость от дозы характерных особенностей (ПМД и ОМД), на распределениях для обоих типов ионов. Как известно, поверхностный максимум дефектов соответствует разупорядоченному слою, который имеет аморфную [9] и(или) нанокристаллическую [12] структуру. На рис. 3 показана дозовая зависимость толщины ПМД для бомбардировки нитрида галлия ионами $\mathrm{F}$ и $\mathrm{Ne}$ с энергией 1.3 кэB/a.e.м. Толщина этого слоя определялась из спектров RBS/C с помощью алгоритма, предложенного в [13] (см. также в [14]). Как видно из рис. 3 , заметных различий в ходе данных зависимостей для обоих ионов в рамках экспериментальных погрешностей не наблюдается, и они могут быть представлены единой кривой. 

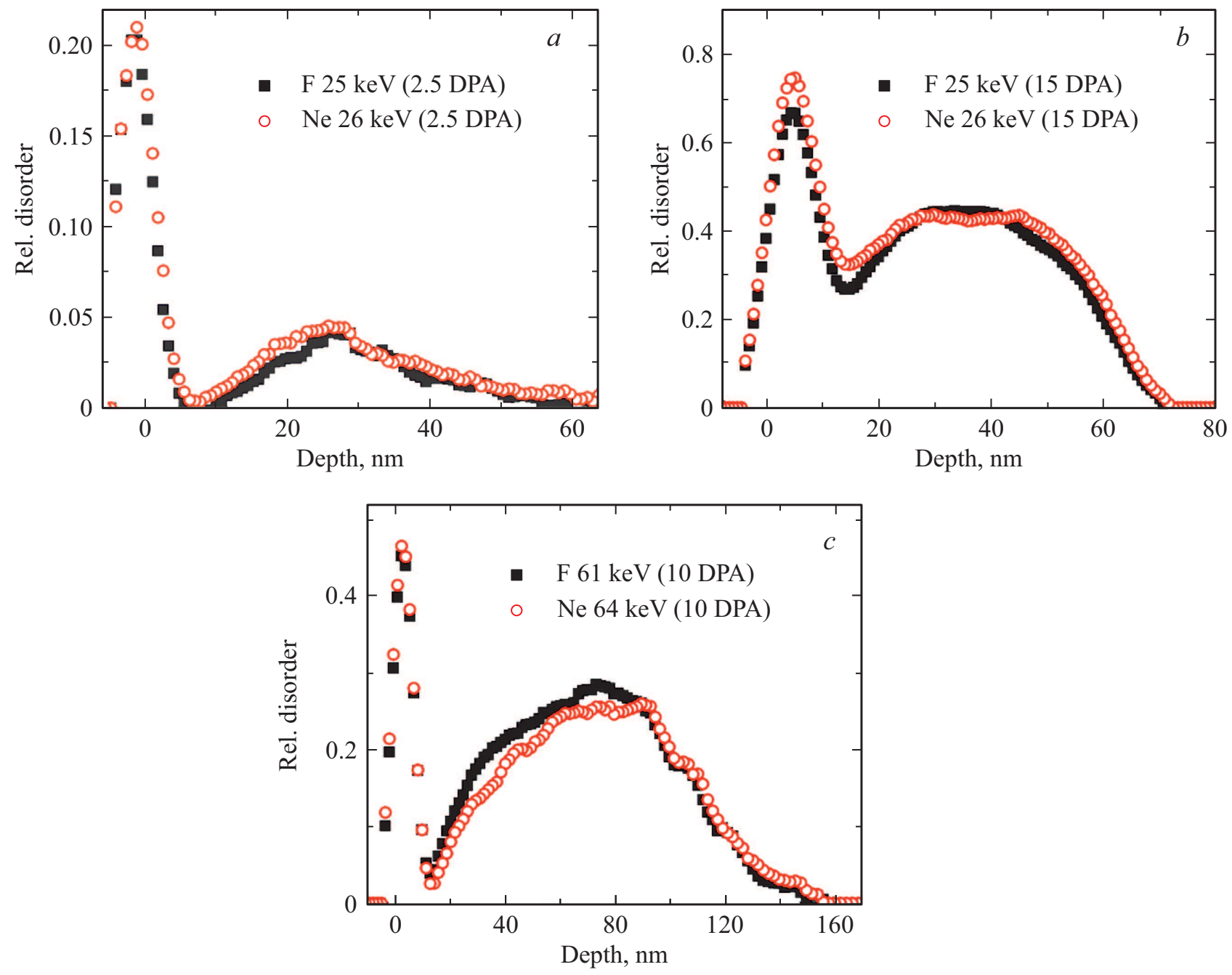

Рис. 2. Распределения структурных нарушений по глубине $\mathrm{GaN}$, облученного ионами $\mathrm{F}$ и Ne при выполнении условий для корректного сравнения: $a-$ с энергией 1.3 кэВ/а.е.м. и дозой $2.5 \mathrm{DPA} ; b-1.3$ кэВ/а.е.м. и $15 \mathrm{DPA} ; c-3.2$ кэB/a.e.м. и $10 \mathrm{DPA}$.

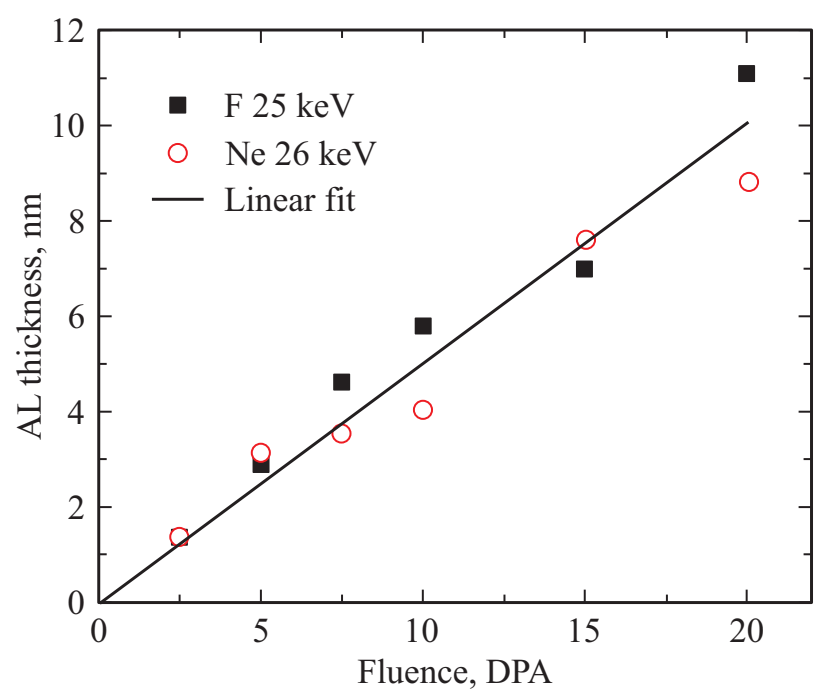

Рис. 3. Зависимость толщины поверхностного слоя $\mathrm{GaN}$ c нарушенной кристаллической структурой от дозы облучения ионами $\mathrm{F}$ и $\mathrm{Ne}$ с энергией 1.3 кэB/a.e.м.
На рис. 4 показан ход роста величины относительного разупорядочения в ОМД с увеличением дозы ионов $\mathrm{F}$ и $\mathrm{Ne}$ с энергиями 1.3 и 3.2 кэВ/а.е.м. при имплантации их в $\mathrm{GaN}$. Опять экспериментальные точки для каждой энергии для обоих ионов ,ложатся“ на одну кривую независимо от их типа. Заметим, что ход такой зависимости аналогичен ходу подобных зависимостей для других ионов $[11,15]$.

Для сравнения на этом же рисунке приведем дозовую зависимость относительного повреждения в максимуме ОМД для случая, когда, наоборот, химические свойства внедряемых ионов заведомо влияют на ход подобного процесса. В [2] было убедительно показано, что сильно выраженный химический эффект имеет место при имплантации в $\mathrm{GaN}$ ионов углерода. На рис. 4 приведена относительная концентрация структурных нарушений углерода с энергией 40 кэВ (3.3 кэВ/а.е.м.) как функция дозы, полученная нами из RBS/C спектров этой работы [2].

Следует отметить, что скорость генерации первичных смещений внедряемыми ионами углерода (см. рис. 1) 


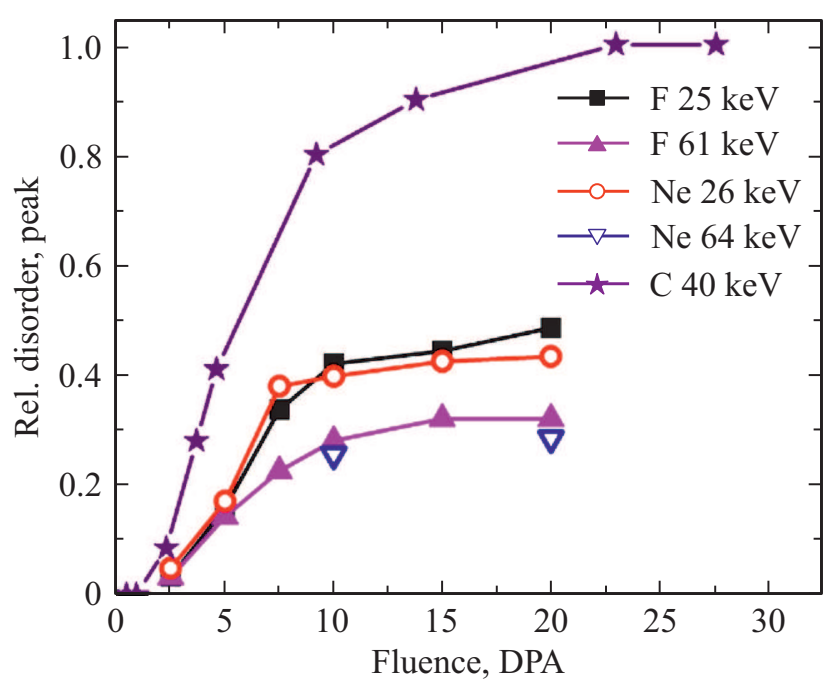

Рис. 4. Дозовые зависимости относительного разупорядочения в объемном максимуме дефектов при облучении $\mathrm{GaN}$ ионами, указанными на вставке. Данные для ионов С c энергией 40 кэВ получены из результатов [2] (подробнее см. в тексте).

была несколько выше, чем при облучении фтором или неоном, поскольку плотность потока ионов углерода $\left(6.45 \cdot 10^{-3} \mathrm{DPA} \cdot \mathrm{c}^{-1}\right)$ несколько превышала таковую для последних $\left(3.6 \cdot 10^{-3} \mathrm{DPA} \cdot \mathrm{c}^{-1}\right)$. Однако столь небольшое различие не могло столь драматически повлиять на ход накопления структурных нарушений в ОМД [16]. Действительно, если для ионов $\mathrm{Ne}$ и $\mathrm{F}$ с ростом дозы имеет место насыщение уровня повреждения ОМД при относительной концентрации дефектов, меньшей 0.5 , то при бомбардировке углеродом происходит полная аморфизация нитрида галлия в области ОМД.

\section{4. Заключение}

Таким образом, из всех полученных данных по накоплению дефектов кристаллической структуры следует, что химические эффекты при внедрении ионов фтора в нитрид галлия не влияют в пределах более $10 \%$ на эффективность накопления структурных нарушений по крайней мере до доз, не превышающих 20 DPA, что соответствует концентрации внедренных при энергии 25 кэВ атомов фтора $\sim 10^{22} \mathrm{~cm}^{-3}$.

\section{Финансирование работы}

Работа выполнена при поддержке Российского фонда фундаментальных исследований, грант РФФИ № 18-08-01213.

\section{Конфликт интересов}

Авторы заявляют, что у них нет конфликта интересов.

\section{Список литературы}

[1] M. Nastasi, J.W. Mayer. Ion Implantation and Synthesis of Materials (Berlin: Springer, 2006).

[2] S.O. Kucheyev, J.S. Williams, C. Jagadish, J. Zou, G. Li. Phys. Rev. B, 62, 7510 (2000).

[3] S.O. Kucheyev, J.S. Williams, C. Jagadish, J. Zou, G. Li, A.I. Titov. Phys. Rev. B, 64, 035202 (2001).

[4] A.I. Titov, P.A. Karaseov, K.V. Karabeshkin, V.S. Belyakov, A.V. Arkhipov, S.O. Kucheyev. Nucl. Instrum. Meth. Phys. Res. B, 315, 257 (2013).

[5] Н.Н. Герасименко, А.А. Жуков, Н.Н. Герасименко (мл.), А.Н. Тарасенков, И.В. Ловягин. Изв. вузов. Электроника, № 4-5, 185 (2005).

[6] S.O. Kucheyev. Ion beam processes group-III nitrides. PhD thesis (The Australian National University, 2002) p. 72.

[7] J.F. Ziegler, J.P. Biersack, U. Littmark. The Stopping and Range of Ions in Solids (Pergamon Press, N.Y., 1985); J.F. Ziegler, SRIM-2013 software package, available online at http://www.srim.org.

[8] K. Schmid. Rad. Effects, 17, 201 (1973).

[9] S.O. Kucheyev, J.S. Williams, S.J. Pearton. Mater. Sci. Eng. R, 33, 51 (2001).

[10] K. Lorenz, U. Wahl, E. Alves, E. Nogales, S. Dalmasso, R.W. Martin, K.P. O’Donnell, M. Wojdak, A. Braud, T. Monteiro, T. Wojtowicz, P. Ruterana, S. Ruffenach, O. Briot. Opt. Mater., 28, 750 (2006).

[11] S.O. Kucheyev, A.Yu. Azarov, A.I. Titov, P.A. Karaseov, T.M. Kuchumova. J. Phys D: Appl. Phys., 42, 085309 (2009).

[12] F. Gloux, T. Wojtowicz, P. Ruterana, K. Lorenz, E. Alves. J. Appl. Phys., 100, 073520 (2006).

[13] А.Ю. Азаров. ФТП, 38, 1445 (2004).

[14] К.В. Карабешкин, П.А. Карасёв, А.И. Титов. ФТП, 50, 1009 (2016).

[15] A.I. Titov, P.A. Karaseov, A.Yu. Kataev, A.Yu. Azarov, S.O. Kucheyev. Nucl. Instrum. Meth. in Phys Res. B, 277, 80 (2012).

[16] S.O. Kucheyev, J. Zou, J.S. Williams, C. Jagadish, G. Li. Nucl. Instrum. Meth. B, 178, 209 (2001).

Редактор Г.А. Оганесян

\section{Do chemical effects enhance damage buildup in GaN during fluorine ion irradiation?}

\section{A.I. Titov, K.V. Karabeshkin, P.A. Karaseov, A.l. Struchkov}

Peter the Great St. Petersburg Polytechnic University, 195251 St. Petersburg, Russia

Abstract Structural damage buildup in $\mathrm{GaN}$ under irradiation by 1.3 and $3.2 \mathrm{keV} / \mathrm{amu} \mathrm{F}$ and $\mathrm{Ne}$ ions has been studied. It is shown that chemical effects during irradiation with fluorine ions do not enhance formation of stable structural damage on the surface or in the bulk of $\mathrm{GaN}$ at all the doses considered. 